\title{
Stellar populations and AGN in the bulges of SDSS galaxies
}

\author{
Vivienne Wild $^{1} \dagger$, Guinevere Kauffmann ${ }^{1}$ and Tim Heckman ${ }^{2}$ \\ ${ }^{1}$ Max-Planck-Institut für Astrophysik, Karl-Schwarzschild Str. 1, 85748 Garching, Germany \\ ${ }^{2}$ Department of Physics and Astronomy, Johns Hopkins University, Baltimore, MD21218, US
}

\begin{abstract}
We present a new method for measuring the recent star formation history (SFH) of galaxies from their $4000 \AA$ break and Balmer absorption line strength. By making use of the whole $3750-4150 \AA$ spectral region, the method achieves a higher signal-to-noise ratio than traditional indices. The new technique is ideally suited to finding evidence for weak or old starbursts in good quality spectra, or studying post-starburst galaxies at high redshift. On application to massive, bulge dominated galaxies in the SDSS, we find clear correlations between the recent star formation history of bulges and the properties of the AGN.
\end{abstract}

Keywords. galaxies: bulges, galaxies: active, methods: statistical

\section{The new method}

We have created new spectral indices designed to measure the recent $(\lesssim 1 \mathrm{Gyr}) \mathrm{SFH}$ of galaxies from their stellar populations. Instead of using restricted wavelength regions around lines of known interest, we utilise the entire wavelength region $3750-4150 \AA$. This allows all of the absorption features, together with the continuum shape, to be used in combination to provide a higher signal-to-noise ratio (SNR) measure of the stellar population. The method is based on a Principal Component Analysis (PCA) of a set of Bruzual \& Charlot population synthesis model spectra (Bruzual \& Charlot 2003). The PCA looks for correlated patterns in the input dataset, and orders these "eigenspectra" by the variance they account for (see Figure 1, left). As intuitively expected, the PCA identifies the $4000 \AA$ break strength as the first component, and combines this with the correlation with Balmer absorption line strength. The second component accounts for an excess in Balmer absorption, combined with a corresponding decrease in strong blue continuum from hot stars. The third component clearly identifies $\mathrm{Ca} I \mathrm{II}(\mathrm{H} \& \mathrm{~K})$ as the third axis of variation in this region of galaxy spectra (see also Leonardi \& Rose 1996). Galaxy spectra can be reconstructed by combining differing amplitudes of these eigenspectra: these amplitudes are our new indices. Plotting the new indices against $\mathrm{D}_{n}(4000)$ and $\mathrm{H} \delta_{\mathrm{A}}$ gives an immediate impression of their equivalence (Figure 1, right). Figure 2 uses duplicate observations of galaxies in the SDSS to show the improvement afforded by measuring Balmer absorption line strength using this method.

\section{Application to the Sloan Digital Sky Survey}

We have applied the new technique to 16606 high stellar surface mass density (bulge dominated) galaxies at $0.01<z<0.07$ in the SDSS. We use the restricted 3" SDSS fibre aperture to our advantage: at these redshifts it corresponds to a diameter of $0.6-4 \mathrm{kpc}$, allowing us to focus on the stellar populations of the galaxy bulges. By being able to

$\dagger$ vwild@mpa-garching.mpg.de 

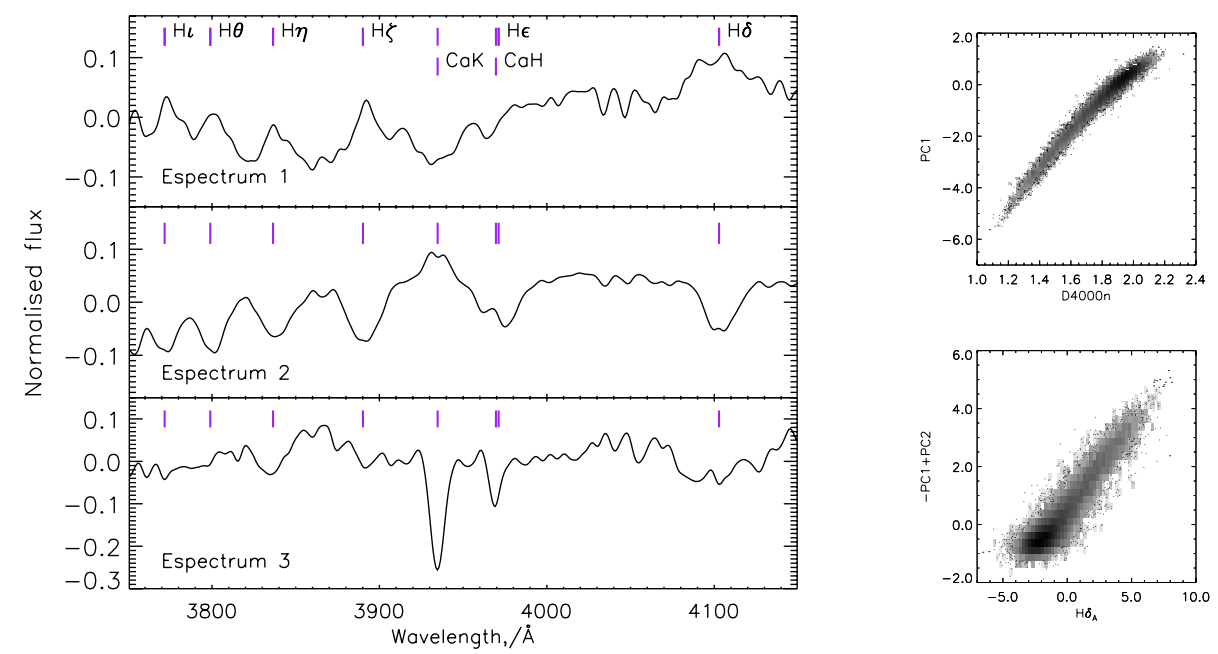

Figure 1. Left: The eigenspectra. Right: Comparing the new indices with traditional indices.
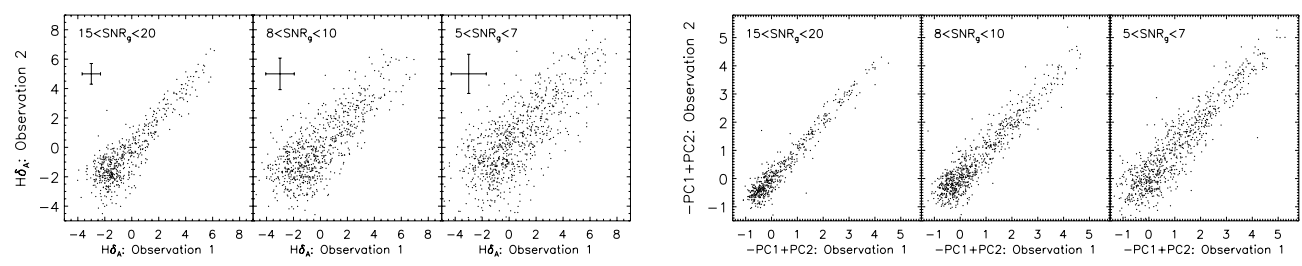

Figure 2. Measuring $\mathrm{H} \delta_{\mathrm{A}}$ and the new indices in duplicate observations of SDSS galaxies as a function of SNR of the spectra demonstrates the dramatic improvement.

characterise the recent SFHs of the bulges to greater accuracy, we are able to build on previous results (e.g. Kauffmann et al. 2003).

The majority $(\sim 70 \%)$ of bulges in the sample are quiescent with no signs of recent or ongoing star formation. Although half of all active galactic nuclei (AGN) lie within these bulges, they contribute only $\sim 5 \%$ to the total black hole growth in the local Universe. At the other extreme, the $\sim 5 \%$ of galaxy bulges that are undergoing or have recently undergone the strongest starbursts contain AGN which contribute $\sim 35 \%$ of the total black hole growth. We conclude that our results support the popular hypothesis for black hole growth occurring through strong gas inflow into the central regions of galaxies, followed by a strong starburst and triggering of the AGN. However, the remainder of the black hole growth $(>50 \%)$ is contributed by galaxy bulges with more moderate recent or ongoing star formation and we conclude that the dominant cause of black hole growth in the local Universe is less spectacular in nature.

\section{Acknowledgements}

VW is supported by the MAGPOP Marie Curie EU Research and Training Network.

\section{References}

Bruzual G., \& Charlot, S., 2003, MNRAS, 344, 1000

Kauffmann, G., Heckman, T. M., Tremonti, C., et al., 2003, MNRAS, 346, 1055

Leonardi, A. J., \& Rose, J. A., 1996, AJ, 111, 182 